\title{
FAMILIES OF SETS OF POSITIVE MEASURE
}

\author{
GRZEGORZ PLEBANEK
}

\begin{abstract}
We present a combinatorial description of those families $\mathscr{P}$ of sets, for which there is a finite measure $\mu$ such that $\inf \{\mu(P): P \in \mathscr{P}\}>0$. This result yields a topological characterization of measure-compactness and Borel measure-compactness. It is also applied to a problem on the existence of regular measure extensions.
\end{abstract}

The main part of this paper deals with the following problem.

Given a family $\mathscr{P}$ of subsets of a certain set $X$, under what conditions does there exist a finite measure $\mu$ defined on some $\sigma$-algebra containing $\mathscr{P}$ such that $\inf \{\mu(P): P \in \mathscr{P}\}>0$ ?

The solution to the "finitely additive" version of this problem has been known since Kelley [11] introduced the notion of intersection numbers in order to characterize Boolean algebras having strictly positive (finitely additive) measures. Kelley's idea was subsequently used to describe compact topological spaces that support Radon measures (cf. [6, Chapter 6]). Casteren [5] applied intersection numbers to a problem on the existence of certain Radon measures in topological spaces. In [18] topological spaces having strictly positive Baire measures are characterized with the help of intersection numbers. This notion appears also in Mägerl-Namioka [14] in the context of separability of spaces of measures.

In $\S 2$ of this paper we introduce a combinatorial condition involving intersection numbers which provides an answer to the question mentioned above. The subject is investigated in the context of measures being regular with respect to a given $\delta$-lattice. This setting is suitable for some applications of the main result. Section 3 contains a characterization of measure-compact and Borel measure-compact topological spaces which seems to answer the problems posed by Wheeler [19] and Gardner-Pfeffer [8]. Next we discuss a problem on the existence of regular measure extensions.

\section{Preliminaries}

We start by introducing some notation and terminology. The set of positive integers is denoted by $\mathbb{N} ; 2^{X}$ stands for the power set of a set $X$. For a sequence $\left\{A_{n}\right\}$ in $2^{X}$, we write $A_{n} \downarrow A$ if $A_{1} \supseteq A_{2} \supseteq \cdots$ and $\bigcap_{n \in \mathbb{N}} A_{n}=A$.

Given $\mathscr{A} \subseteq 2^{X}$, the algebra and the $\sigma$-algebra generated by $\mathscr{A}$ are denoted by $a(\mathscr{A})$ and $\sigma(\mathscr{A})$, respectively.

A lattice in $X$ is a family of subsets of $X$ containing $\varnothing, X$ and is closed

Received by the editors February 10, 1989 and, in revised form, March 26, 1990.

1980 Mathematics Subject Classification (1985 Revision). Primary 28A12; Secondary 28C15. 
under finite unions and intersections. A $\delta$-lattice is a lattice closed under countable intersections.

A subfamily $\mathscr{D}$ of a lattice $\mathscr{L}$ is called an $\mathscr{L}$-filter if the following are satisfied:

(i) $X \in \mathscr{D}, \varnothing \notin \mathscr{D}$;

(ii) if $C, D \in \mathscr{D}$ then $C \cap D \in \mathscr{D}$;

(iii) if $D \in \mathscr{D}, L \in \mathscr{L}$, and $D \subseteq L$ then $L \in \mathscr{D}$.

An $\mathscr{L}$-filter is $\sigma$-complete if it is closed under countable intersections. An $\mathscr{L}$-filter is called fixed if its intersection is nonempty. An $\mathscr{L}$-ultrafilter is an $\mathscr{L}$-filter being maximal in the family of all $\mathscr{L}$-filters.

A nonnegative finitely additive set function defined on an algebra of sets is called a quasi-measure. A measure is a countably additive quasi-measure defined on a $\sigma$-algebra.

All measures considered here are assumed to be finite.

A quasi-measure $m$ defined on $\mathscr{A}$ is said to be regular with respect to a lattice $\mathscr{L} \subseteq \mathscr{A}$, or $\mathscr{L}$-regular, if for every $A \in \mathscr{A}$,

$$
m(A)=\sup \{m(L): L \in \mathscr{L}, L \subseteq A\} .
$$

Let $\varphi$ be a quasi-measure on an algebra $\mathscr{A}$ and let $\mathscr{P}$ be any subfamily of $\mathscr{A}$. We use the notation

$$
\inf \varphi(\mathscr{P})=\inf \{\varphi(P): P \in \mathscr{P}\},
$$

for simplicity. The symbol $\left.\varphi\right|_{\mathscr{P}}$ denotes the restriction of $\varphi$ to $\mathscr{P}$ (thus, for example, $\left.\varphi\right|_{\mathscr{P}} \geq \alpha$ means $\varphi(P) \geq \alpha$ for every $\left.P \in \mathscr{P}\right)$.

Now we shall recall the definition of the intersection number of a given family $\mathscr{P} \subseteq 2^{X}$. For a sequence $A_{1}, \ldots, A_{n}$ of not necessarily distinct elements of $\mathscr{P}$ put

$$
i\left(A_{1}, \ldots, A_{n}\right)=\left\|\sum_{k=1}^{n} \chi_{A_{k}}\right\| / n,
$$

where $\|\cdot\|$ is the supremum norm and $\chi_{A}$ denotes the characteristic function of a set $A$. The intersection number of $\mathscr{P}$, denoted by $I(\mathscr{P})$, is defined as follows:

$$
I(\mathscr{P})=\inf \left\{i\left(A_{1}, \ldots, A_{n}\right): A_{k} \in \mathscr{P}, n \geq 1\right\} .
$$

Note that $\left\|\sum_{k=1}^{n} \chi_{A_{k}}\right\|$ is the largest number $N$ such that there is $b \subseteq$ $\{1, \ldots, n\}$ having $N$ elements with $\bigcap_{k \in b} A_{k} \neq \varnothing$. Thus the condition $I(\mathscr{P})>$ 0 expresses a certain combinatorial property of $\mathscr{P}$ which seems to be a finite version of a caliber-type condition (cf. [6, Chapter 6]). A family satisfying it is called a positive family. Clearly every $\mathscr{L}$-filter, where $\mathscr{L}$ is a lattice of sets, is a family with the intersection number 1 .

It follows from the definition that $I(\mathscr{P})=0$ if $\varnothing \in \mathscr{P}$. Note also that if $\mathscr{T} \subseteq \mathscr{P}$ then $I(\mathscr{T}) \geq I(\mathscr{P})$. Given $A \subseteq X$, we write $I(\mathscr{P}, A)$ for the intersection number of the family $\mathscr{P} \cup\{A\}$.

The following result is basic for our considerations.

Theorem $\mathbf{K}$ (Kelley). Let $\mathscr{P} \subseteq 2^{X}$ be given and let $\alpha$ be a real number. The condition $I(\mathscr{P}) \geq \alpha$ is necessary and sufficient for the existence of a probability quasi-measure $m$ on $2^{X}$ such that $\left.m\right|_{\mathscr{P}} \geq \alpha$. Consequently, $I(\mathscr{P})=$ 
sup inf $\varphi(\mathscr{P})$, where the supremum is taken over all probability quasi-measures $\varphi$ on $2^{X}$.

Theorem K comes from [11] (see Theorem 2 and Corollary 3). Wilhelm [20] gives a generalization of this result (together with a more elementary proof).

\section{STRICTLY POSITIVE FAMILIES AND MEASURES}

Throughout this section $X$ is a nonempty space and $\mathscr{L}$ is a $\delta$-lattice in $X$. The letter $L$ (with possible indices) always stands for a member of $\mathscr{L}$.

We start by introducing our basic definition.

Definition 2.1. We say that a subfamily $\mathscr{P}$ of $\mathscr{L}$ is $\mathscr{L}$-strongly positive if

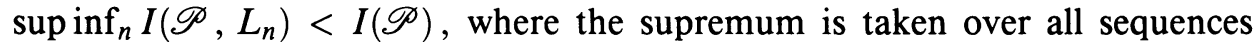
$\left\{L_{n}\right\} \subseteq \mathscr{L}$ with $L_{n} \downarrow \varnothing$.

In other words, $\mathscr{P}$ is $\mathscr{L}$-strongly positive if and only if there exists $\delta<I(\mathscr{P})$ such that for every sequence $\left\{L_{n}\right\} \subseteq \mathscr{L}$, if $L_{n} \downarrow \varnothing$ then $I\left(\mathscr{P}, L_{n}\right)<\delta$ for some $n \in \mathbb{N}$.

The collection of all $\mathscr{L}$-strongly positive subfamilies of $\mathscr{L}$ will be denoted by $\operatorname{sp}(\mathscr{L})$. Furthermore we put

$$
\operatorname{sp}^{*}(\mathscr{L})=\{\mathscr{P}:(\exists \mathscr{T} \in \operatorname{sp}(\mathscr{L})) \mathscr{P} \subseteq \mathscr{T}\}
$$

Having the $\delta$-lattice $\mathscr{L}$ fixed, in this section we often omit " $\mathscr{L}$ " from the phrases " $\mathscr{L}$-strongly positive" and " $\mathscr{L}$-regular."

Observe that if $\mathscr{P}$ is strongly positive then $I(\mathscr{P})>I(\mathscr{P}, \varnothing)=0$; i.e. $\mathscr{P}$ is a positive family. The converse holds if $\mathscr{L}$ is monocompact, i.e. if every $\left\{L_{n}\right\} \subseteq \mathscr{L}$ with $L_{n} \downarrow \varnothing$ contains the empty set.

If $\mathscr{F}$ is $\sigma$-complete $\mathscr{L}$-ultrafilter then $\mathscr{F} \in \operatorname{sp}(\mathscr{L})$, for if $L_{n} \downarrow \varnothing$ then $L_{n_{0}} \notin \mathscr{F}$ for some $n_{0} \in \mathbb{N}$, and $I\left(\mathscr{F}, L_{n_{0}}\right)=1 / 2<1=I(\mathscr{F})$. We shall see later that there is a correspondence between strongly positive families and measures, similar to that linking $\sigma$-complete ultrafilters and $0-1$ measures.

Now we shall prove several technical lemmas leading to the main result of this section, which is stated below as Theorem 2.9.

Lemma 2.2. Let two nonempty families $\mathscr{R}, \mathscr{T} \subseteq 2^{X}$ be given. Assume that for every $R \in \mathscr{R}$ and $T \in \mathscr{T}$ there are $R^{\prime} \in \mathscr{R}$ and $T^{\prime} \in \mathscr{T}$ such that $R^{\prime} \subseteq R$, $T^{\prime} \subseteq T$, and $R^{\prime} \cap T^{\prime}=\varnothing$.

$$
\text { If } I(\mathscr{R})=\alpha>0 \text { and } I(\mathscr{T})=\beta>0 \text { then } I(\mathscr{R} \cup \mathscr{T})=\frac{\alpha \beta}{\alpha+\beta} \text {. }
$$

Proof. We shall use the equivalence given in Theorem $\mathrm{K}$.

There exist probability quasi-measures $\varphi$ and $\psi$ on $2^{X}$ with $\left.\varphi\right|_{\mathscr{R}} \geq \alpha$ and $\left.\psi\right|_{\mathscr{T}} \geq \beta$. Since $m=\frac{\beta}{\alpha+\beta} \varphi+\frac{\alpha}{\alpha+\beta} \psi$ is a probability quasi-measure with $\left.m\right|_{\mathscr{R} \cup \mathscr{T}} \geq \frac{\alpha \beta}{\alpha+\beta}$, it follows that $I(\mathscr{R} \cup \mathscr{T}) \geq \frac{\alpha \beta}{\alpha+\beta}$.

Let $m$ be a probability quasi-measure on $2^{X}$. Take any $a>\alpha$ and $b>\beta$, and choose finite subfamilies $\mathscr{R}_{0} \subseteq \mathscr{R}, \mathscr{T}_{0} \subseteq \mathscr{T}$ such that $I\left(\mathscr{R}_{0}\right)<a$ and $I\left(\mathscr{T}_{0}\right)<b$. Put $X_{1}=\bigcup \mathscr{R}_{0}, X_{2}=\bigcup \mathscr{T}_{0} ;$ by the assumption on $\mathscr{R}$ and $\mathscr{T}$ we can additionally assume $X_{1} \cap X_{2}=\varnothing$. Since $m\left(X_{1}\right)+m\left(X_{2}\right)=m\left(X_{1} \cup X_{2}\right) \leq 1$, we have $m\left(X_{1}\right) \leq \frac{b}{a+b}$ or $m\left(X_{2}\right) \leq \frac{a}{a+b}$. Suppose that the first inequality holds (the second case is similar). There is $R_{0} \in \mathscr{R}$ such that $m\left(R_{0}\right) \leq \operatorname{am}\left(X_{1}\right)$. 
Indeed, otherwise we would have $m\left(X_{1}\right)>0$ and

$$
\left.\frac{1}{m\left(X_{1}\right)} m\right|_{\mathscr{R}_{0}} \geq a>I\left(\mathscr{R}_{0}\right)
$$

a contradiction.

Since $m\left(R_{0}\right) \leq a m\left(X_{1}\right) \leq \frac{a b}{a+b}$, inf $m(\mathscr{R} \cup \mathscr{T}) \leq \frac{a b}{a+b}$. Thus, using Theorem $\mathrm{K}$, we conclude that $I(\mathscr{R} \cup \mathscr{T}) \leq \frac{\alpha \beta}{\alpha+\beta}$.

Lemma 2.3. Let $(X, \mathscr{A}, \mu)$ be a measure space with $\mu$ being nonatomic and $\mathscr{L}$-regular, where $\mathscr{L} \subseteq \mathscr{A}$. Given $A \in \mathscr{A}$ and $t \in[0, \mu(A))$, there is $L \subseteq A$ such that $\mu(L)=t$.

Proof. It is well known (cf. [9, Exercise (2), p. 174] or [8, Lemma 9.1]) that for every $t \in[0, \mu(A))$ there is $B \in \mathscr{A}$ such that $B \subseteq A$ and $\mu(B)=t$. We can easily define $B_{1} \supseteq L_{1} \supseteq B_{2} \supseteq L_{2} \supseteq \cdots$ with $B_{n} \in \mathscr{A}$ and $t<\mu\left(B_{n}\right)<t+1 / n$. Then $L=\bigcap_{n \in \mathbb{N}} L_{n}$ is as required.

Lemma 2.4. Let $\mu$ be a probability measure on $\sigma(\mathscr{L})$, which is nonatomic and $\mathscr{L}$-regular. For a real number $\alpha$ put $\mathscr{P}_{\alpha}=\{L: \mu(L) \geq \alpha\}$.

(a) $I\left(\mathscr{P}_{\alpha}\right)=\alpha$ for every $\alpha \in[0,1]$;

(b) if $\alpha \in(0,1)$ then $\inf _{n} I\left(\mathscr{P}_{\alpha}, L_{n}\right) \leq \frac{\alpha}{1+\alpha}$ whenever $L_{n} \downarrow \varnothing$.

Proof. (a) $I\left(\mathscr{P}_{\alpha}\right) \geq \alpha$ by Theorem $\mathrm{K}$ and the equality holds if $\alpha=1$. Suppose $\alpha<1$; for every $n \in \mathbb{N}$ choose $k_{n} \in \mathbb{N}$ such that

$$
\frac{k_{n}}{n+1} \geq \alpha>\frac{k_{n}-1}{n+1} \text {. }
$$

By Lemma 2.3 there are disjoint sets $L_{1}, \ldots, L_{n}$ of measure $\frac{1}{n+1}$. Let $\pi$ denote the family of all subsets of $\{1, \ldots, n\}$ having $k_{n}$ elements (note that $k_{n} \leq n$ for large $n$ 's). For every $\gamma \in \pi$ we put $L_{\gamma}=\bigcup_{j \in \gamma} L_{j} ; L_{\gamma} \in \mathscr{P}_{\alpha}$ since $\mu\left(L_{\gamma}\right)=k_{n} /(n+1) \geq \alpha$. Now consider $g=\sum_{\gamma \in \pi} \chi_{L_{\gamma}}$.

$$
\frac{\|g\|}{\operatorname{card} \pi}=\left(\begin{array}{c}
n-1 \\
k_{n}-1
\end{array}\right) /\left(\begin{array}{c}
n \\
k_{n}
\end{array}\right)=\frac{k_{n}}{n}<\frac{\alpha(n+1)+1}{n}=\alpha+\frac{\alpha+1}{n} .
$$

Therefore $I\left(\mathscr{P}_{\alpha}\right) \leq \alpha+\frac{\alpha+1}{n}$, and consequently $I\left(\mathscr{P}_{\alpha}\right) \leq \alpha$.

(b) First consider fixed $L_{0} \neq \varnothing$ such that $\mu\left(L_{0}\right)<1-\alpha$. Let $\mathscr{T}=\{L \in$ $\left.\mathscr{P}_{\alpha}: L \cap L_{0}=\varnothing\right\}$; we know from part (a) that $I(\mathscr{T})=\alpha /\left(1-\mu\left(L_{0}\right)\right)$. Applying Lemma 2.2 to the families $\mathscr{T}$ and $\left\{L_{0}\right\}$ we get

$$
I\left(\mathscr{P}_{\alpha}, L_{0}\right) \leq I\left(\mathscr{T}, L_{0}\right) \leq \frac{\alpha}{1-\mu\left(L_{0}\right)}:\left(\frac{\alpha}{1-\mu\left(L_{0}\right)}+1\right)=\frac{\alpha}{1-\mu\left(L_{0}\right)+\alpha} .
$$

Now take a sequence $\left\{L_{n}\right\}$ decreasing to the empty set; since $\lim \mu\left(L_{n}\right)=0$ and $\alpha<1$, we have $\mu\left(L_{n}\right)<1-\alpha$ for large $n$ 's. It follows from the above observation that

$$
\inf _{n} I\left(\mathscr{P}_{\alpha}, L_{n}\right) \leq \inf _{n} \frac{\alpha}{1-\mu\left(L_{n}\right)+\alpha}=\frac{\alpha}{1+\alpha}
$$

Proposition 2.5. Let $\mu$ be an $\mathscr{L}$-regular probability measure defined on $\sigma(\mathscr{L})$.

(a) If $\mu$ is nonatomic then the family $\mathscr{P}_{\alpha}=\{L: \mu(L) \geq \alpha\}$ is $\mathscr{L}$-strongly positive for every $\alpha \in(0,1)$; 
(b) if $T_{1}, \ldots, T_{r}$ are atoms of $\mu$ then the family

$$
\mathscr{T}=\left\{L:(\exists k \leq r) \mu\left(T_{k} \cap L\right)=\mu\left(T_{k}\right)\right\}
$$

is $\mathscr{L}$-strongly positive.

Proof. (a) Using Lemma 2.4 we get

$$
\inf _{n} I\left(\mathscr{P}_{\alpha}, L_{n}\right) \leq \frac{\alpha}{\alpha+1}<\alpha=I\left(\mathscr{P}_{\alpha}\right),
$$

whenever $L_{n} \downarrow \varnothing$, which means that $\mathscr{P}_{\alpha}$ is strongly positive.

(b) We can assume that the sets $T_{k}$ 's are pairwise disjoint. It is easy to check that $I(\mathscr{T})=1 / r$. If $L_{n} \downarrow \varnothing$ then there is $n_{0} \in \mathbb{N}$ such that $\mu\left(L_{n_{0}} \cap T_{k}\right)=0$ for every $k \leq r$. Since the set $T_{k}-L_{n_{0}}$ is an atom, there is $S_{k} \in \mathscr{L}$ with $S_{k} \subseteq T_{k}-L_{n_{0}}$ and $\mu\left(S_{k}\right)=\mu\left(T_{k}\right), k=1, \ldots, r$. Hence

$$
I\left(\mathscr{T}, L_{n_{0}}\right) \leq i\left(S_{1}, \ldots, S_{r}, L_{n_{0}}\right)=1 /(r+1)<1 / r,
$$

which proves that $\mathscr{T}$ is strongly positive.

Proposition 2.6. If $\mu$ is an $\mathscr{L}$-regular probability measure on $\sigma(\mathscr{L})$ then the family $\mathscr{P}_{\alpha}=\{L: \mu(L) \geq \alpha\}$ belongs to $\mathbf{s p}^{*}(\mathscr{L})$ for every $\alpha \in(0,1]$.

Proof. Write $\mu=\mu_{a}+\mu_{c}$, where $\mu_{a}$ is purely atomic and $\mu_{c}$ is nonatomic. If $\mu_{a}=0$ then there is nothing to be done in view of Proposition 2.5(a); suppose $\mu_{a}>0$ and take atoms $T_{1}, \ldots, T_{r}$ such that $\mu_{a}\left(X-\left(T_{1} \cup \cdots \cup T_{r}\right)\right)<\alpha / 2$. Let $\mathscr{T}$ be as in Proposition 2.5(b), i.e. $\mathscr{T}=\left\{L:(\exists k \leq r) \mu\left(L \cap T_{k}\right)=\mu\left(T_{k}\right)\right\}$. If $\mu_{c}=0$ then $\mathscr{P}_{\alpha} \subseteq \mathscr{T}$ and we are done. Otherwise the family $\mathscr{R}=\left\{L: \mu_{c}(L) \geq\right.$ $\left.\alpha / 2 \mu_{c}(X)\right\}$ is strongly positive by Proposition 2.5(a), so there is $\delta<I(\mathscr{R})$ such that $\inf _{n} I\left(\mathscr{R}, L_{n}\right)<\delta$ for every $L_{n} \downarrow \varnothing$. Observe that $\mathscr{P}_{\alpha} \subseteq \mathscr{R} \cup \mathscr{T}$; indeed if $L \in \mathscr{P}_{\alpha}-\mathscr{T}$ then

$$
\mu_{c}(L)=\mu(L)-\mu_{a}(L) \geq \alpha-\frac{\alpha}{2}=\frac{\alpha}{2} \geq \frac{\alpha}{2} \mu_{c}(X),
$$

so $L \in \mathscr{R}$. Thus it is enough to check that the family $\mathscr{R} \cup \mathscr{T}$ is strongly positive.

Denote $I(\mathscr{R})=a, I(\mathscr{T})=b$, and $\Delta=\frac{\delta b}{\delta+b}$. Then $\Delta<\frac{a b}{a+b}=I(\mathscr{R} \cup \mathscr{T})$ (the last equality holds by Lemma 2.2). Let $\left\{L_{n}\right\}$ be a sequence with $L_{n} \downarrow \varnothing$. There is $n_{0} \in \mathbb{N}$ such that $L_{n_{0}} \notin \mathscr{T}$ and $I\left(\mathscr{R}, L_{n_{0}}\right)<\delta$. Applying Lemma 2.2 to the families $\mathscr{R} \cup\left\{L_{n_{0}}\right\}$ and $\mathscr{T}$ we get

$$
I\left(\mathscr{R} \cup \mathscr{T}, L_{n_{0}}\right)=I\left(\mathscr{R} \cup\left\{L_{n_{0}}\right\} \cup \mathscr{T}\right)<\frac{\delta b}{\delta+b}=\Delta .
$$

Hence $\mathscr{R} \cup \mathscr{T}$ is strongly positive and this finishes the proof.

We can obtain a kind of converse to Proposition 2.6 based on the following result, which indeed is true for an arbitrary lattice $\mathscr{L}$.

Given a probability quasi-measure $\varphi$ on a(L), there exists an $\mathscr{L}$-regular probability quasi-measure $m$ on a $\mathscr{L})$ with $\left.m\right|_{\mathscr{L}} \geq\left.\varphi\right|_{\mathscr{L}}$.

This is due to Lembcke [13, Korollar 2.12]; see also [17, Proposition 3.4].

Proposition 2.7. If $\mathscr{P}$ is $\mathscr{L}$-strongly positive then there exists an $\mathscr{L}$-regular measure $\mu$ defined on $\sigma(\mathscr{L})$ such that $\inf \mu(\mathscr{P})>0$.

Proof. Put $\alpha=I(\mathscr{P})>0$ and let $\delta<\alpha$ be a constant as in the remark after Definition 2.1. By Theorem K there is a probability quasi-measure $\varphi$ on 
$a(\mathscr{L})$ with $\left.\varphi\right|_{\mathscr{D}} \geq \alpha$. Let $m$ be a regular probability quasi-measure such that $\left.m\right|_{\mathscr{L}} \geq\left.\varphi\right|_{\mathscr{L}}$; consider the Yosida-Hewitt decomposition of $m$, say $m=\mu+m_{p}$, where $\mu$ is countably additive and $m_{p}$ is purely finitely additive.

We shall check that $m(L)<\delta$ whenever $I(\mathscr{P}, L)<\delta$. The inequality $I(\mathscr{P}, L)<\delta$ means that there are $n, k \in \mathbb{N}$ and $P_{1}, \ldots, P_{n} \in \mathscr{P}$ such that letting $g=\sum_{j \leq n} \chi_{P_{j}}+k \chi_{L}$ we have $\|g\|<(n+k) \delta$. Then

$$
\begin{aligned}
n \alpha+k m(L) & \leq \sum_{j \leq n} m\left(P_{j}\right)+k m(L) \\
& =\int g d m \leq\|g\|<(n+k) \delta<n \alpha+k \delta,
\end{aligned}
$$

and consequently $m(L)<\delta$.

Thus $\lim m\left(L_{n}\right)<\delta$ for every sequence $\left\{L_{n}\right\} \subseteq \mathscr{L}$ decreasing to the empty set. Employing the regularity of $m$ and the formula for $m_{p}$ (cf. [4, Theorem 10.22]), we obtain

$$
\begin{aligned}
m_{p}(X) & =\sup \left\{\lim m\left(A_{n}\right): A_{n} \in a(\mathscr{L}), A_{n} \downarrow \varnothing\right\} \\
& =\sup \left\{\lim m\left(L_{n}\right): L_{n} \downarrow \varnothing\right\} \leq \delta .
\end{aligned}
$$

Hence $\mu(P)=m(P)-m_{p}(P) \geq \varphi(P)-m_{p}(X) \geq \alpha-\delta>0$ for every $P \in \mathscr{P}$. Being countably additive and $\mathscr{L}$-regular, $\mu$ has an extension to an $\mathscr{L}$-regular measure on $\sigma(\mathscr{L})$ (cf. [16, 3(i)]); the proof is then complete.

Note that in Lemma 2.7 we cannot demand inf $\mu(\mathscr{P}) \geq I(\mathscr{P})$ together with $\mu(X)=1$ as the following example shows.

Example 2.8. Let $\mathscr{P} \subseteq 2^{[0,1]}$ be the family of all $P$ that can be written as $P=(U \cap \mathbb{Q}) \cup B$, where $U \subseteq[0,1 / 4]$ is open, $B \subseteq[1 / 4,1], B \in \mathscr{B}$, and $\lambda(U \cup B) \geq 1 / 2$ (here $\mathbb{Q}$ is the set of rationals, $\mathscr{B}$ is the Borel $\sigma$-algebra in $[0,1], \lambda$ denotes the Lebesgue measure). Letting $\mathscr{T}=\{B \in \mathscr{B}: B \subseteq$ $[1 / 4,1], \lambda(B) \geq 1 / 2\}$ we find that $I(\mathscr{P})=1 / 2, I(\mathscr{T})=2 / 3, \mathscr{T}$ being $\mathscr{B}$ strongly positive. If $\left\{A_{n}\right\} \subseteq \mathscr{B}$ with $A_{n} \downarrow \varnothing$ then (applying Lemma 2.4(b)),

$$
\inf _{n} I\left(\mathscr{P}, A_{n}\right) \leq \lim I\left(\mathscr{T}, A_{n}\right)=\frac{\frac{2}{3}}{1+\frac{2}{3}}=\frac{2}{5}<\frac{1}{2}=I(\mathscr{P}) \text {. }
$$

Hence $\mathscr{P}$ is strongly positive. Suppose $\mu$ is a Borel probability with $\left.\mu\right|_{\mathscr{P}} \geq$ $1 / 2$. Let $U_{n}=(0,1 / 4) \backslash\left\{q_{1}, \ldots, q_{n}\right\}$, where $\mathbb{Q}=\left\{q_{k}: k \in \mathbb{N}\right\}$. For every $1 \leq j \leq 3$ we have $P_{n}^{j}=\left(U_{n} \cap \mathbb{Q}\right) \cup[j / 4,(j+1) / 4] \in \mathscr{P}$ and $\mu([j / 4,(j+1) / 4])$ $=\mu\left(\bigcap_{n} P_{n}^{j}\right) \geq 1 / 2$. This means that $\mu([0,1]) \geq 3 / 2$, a contradiction.

Theorem 2.9. Let $\mathscr{P}$ be a subfamily of a $\delta$-lattice $\mathscr{L}$.

(a) There exist an $\mathscr{L}$-regular probability measure $\mu$ on $\sigma(\mathscr{L})$ such that $\inf \mu(\mathscr{P})>0$ if and only if $\mathscr{P} \in \mathbf{s p}^{*}(\mathscr{L})$

(b) there exists an $\mathscr{L}$-regular probability measure $\mu$ on $\sigma(\mathscr{L})$ being positive on elements of $\mathscr{P}$ if and only if $\mathscr{P}$ can be written as $\mathscr{P}=\bigcup_{n \in \mathbb{N}} \mathscr{P}_{n}$, where $\mathscr{P}_{n} \in \mathbf{s p}^{*}(\mathscr{L})$ for every $n$.

Proof. Part (a) follows immediately from Proposition 2.6 and Proposition 2.7. If $\mu$ is regular and $\mu(P)>0$ for every $P \in \mathscr{P}$ then putting $\mathscr{P}_{n}=\{P \in$ $\mathscr{P}: \mu(P) \geq 1 / n\}$ we know from Lemma 2.6 that $\mathscr{P}_{n} \in \mathrm{sp}^{*}(\mathscr{L})$, and the decomposition holds. On the other hand, if $\mathscr{P}_{n} \in \mathrm{sp}^{*}(\mathscr{L})$ for every $n$ then choose 
regular probability measures $\mu_{n}$ with $\left.\mu_{n}\right|_{\mathscr{P}_{n}}>0$. Then $\mu=\sum_{n \in \mathbb{N}} 2^{-n} \mu_{n}$ is as required.

Corollary 2.10. For an $\mathscr{L}$-filter $\mathscr{D}$, the condition $\mathscr{D} \in \operatorname{sp}^{*}(\mathscr{L})$ is necessary and sufficient for the existence of a probability measure $\mu$ satisfying $\mu(D)=1$, all $D \in \mathscr{D}$.

Proof. If such $\mu$ exists then $\mathscr{D} \subseteq\{L: \mu(L)=1\} \in \operatorname{sp}^{*}(\mathscr{L})$ by Lemma 2.6. To prove the reverse implication, let $\nu$ be a regular measure with $\left.\nu\right|_{\mathscr{D}} \geq \delta$, where $\delta>0$ (Lemma 2.7). For $A \in \mathscr{A}=\sigma(\mathscr{L})$ we define

$$
\mu(A)=\inf \{\nu(A \cap D): D \in \mathscr{D}\} .
$$

A standard argument shows that $\mu$ is a quasi-measure; since $\mu \leq \nu, \mu$ is a regular measure on the $\sigma$-algebra $\mathscr{A}$. Note that $\mu(D)=\mu(X)>0$ for every $D \in \mathscr{D}$. Thus the measure $\mu / \mu(X)$ is as required.

Corollary 2.10 may be compared with the following known result (cf. [2]).

There exists an $\mathscr{L}$-regular $0-1$ measure $\mu$ such that $\left.\mu\right|_{\mathscr{D}}=1$ if and only if $\mathscr{D}$ is contained in a $\sigma$-complete $\mathscr{L}$-ultrafilter.

We end this section with an example showing that if the supremum were omitted from the definition of a strongly positive family we would get an essentially weaker property.

Example 2.11. Let $X=[0,2]$ and let $\mathscr{B}$ be the Borel $\sigma$-algebra of $X$. We shall prove that there exists a family $\mathscr{P} \subseteq \mathscr{B}$ such that $\mathscr{P} \notin$ sp* $\left.^{*} \mathscr{B}\right)$ but inf $I\left(\mathscr{P}, B_{n}\right)<I(\mathscr{P})$ whenever $\left\{B_{n}\right\} \subseteq \mathscr{B}$ is such that $B_{n} \downarrow \varnothing$.

Put $I_{0}=[0,1]$ and $I_{1}=[1,2]$. Let $\mathscr{F}$ be the algebra in $I_{0}$ generated by intervals and let $\mathscr{M}$ be the family of meager subsets of $I_{0}$. Denoting by $\lambda$ the Lebesgue measure on the real line we put $\varphi(F-M)=\lambda(F)$ for every $F \in \mathscr{F}$ and $M \in \mathscr{M}$. This uniquely defines a probability quasi-measure on the algebra $a(\mathscr{F} \cup \mathscr{M})$; let $\psi$ be any quasi-measure on $\mathscr{B}$ extending $\varphi$. Note that if $\psi(B)=1$ then $I_{0}-B \in \mathscr{M}$. Now consider the family $\mathscr{P}=\{B \in \mathscr{B}$ : $m(B) \geq 1 / 2\}$, where $m(B)=\frac{1}{2}\left(\psi\left(B \cap I_{0}\right)+\lambda\left(B \cap I_{1}\right)\right)$. Clearly $I(\mathscr{P})=1 / 2$; we have to know that $m(B) \geq 1 / 2$ for every $B \in \mathscr{B}$ with $I(\mathscr{P}, B)=1 / 2$. This can be checked as in the proof of Lemma 2.4 (although $m$ is not $\sigma$-additive, it has the property stated in Lemma 2.3 and the same argument works).

Now take a sequence $\left\{B_{n}\right\} \subseteq \mathscr{B}$ such that $B_{n} \downarrow \varnothing$. Then there is $j$ such that $I_{0}-B_{j}$ is not meager; consequently $\psi\left(I_{0} \cap B_{j}\right)<1$. Take $k>j$ such that $\lambda\left(B_{k} \cap I_{1}\right)<1-\psi\left(B_{j} \cap I_{0}\right)$. Thus

$$
m\left(B_{k}\right) \leq \frac{1}{2}\left(\psi\left(B_{j} \cap I_{0}\right)+\lambda\left(B_{k} \cap I_{1}\right)\right)<1 / 2,
$$

and therefore $I\left(\mathscr{P}, B_{k}\right)<1 / 2$.

On the other hand, $\mathscr{P} \notin \mathrm{sp}^{*}(\mathscr{B})$. Otherwise there exists a Borel measure $\mu$ in $X$ such that inf $\mu(\mathscr{P})>0$. In particular, $\mu\left(I_{0}-M\right)>0$ for every $M \in \mathscr{M}$, a contradiction, since every Borel measure in $I_{0}$ is concentrated on a meager set (cf. [15, Théorème 1]).

\section{ON MEASURE-COMPACT AND BOREL MEASURE-COMPACT SPACES}

In this section we present a characterization of measure-compact and Borel measure-compact topological spaces. We begin with establishing some notation and terminology concerning measures in topological spaces. 
Topological spaces are always assumed to be Hausdorff. The families of zero sets and closed sets in a space $X$ are denoted by $\mathscr{Z}_{X}$ and $\mathscr{F}_{X}$, respectively. A Baire measure (Borel measure) in $X$ is a finite measure defined on $\sigma\left(\mathscr{Z}_{X}\right)$ (on $\left.\sigma\left(\mathscr{F}_{X}\right)\right)$. A Borel measure being $\mathscr{F}_{X}$-regular is called a regular Borel measure.

A nonempty family of sets $\mathscr{D}$ is said to be directed downwards if for every $D_{1}, D_{2} \in \mathscr{D}$ there is $D_{3} \in \mathscr{D}$ with $D_{3} \subseteq D_{1} \cap D_{2}$. We write $\mathscr{D} \downarrow D$ if $\mathscr{D}$ is directed downwards and $D=\bigcap \mathscr{D}$.

A Baire measure $\mu$ in $X$ is called $\tau$-additive if $\inf \mu(\mathscr{D})=0$ for every family $\mathscr{D} \subseteq \mathscr{Z}_{X}$ with $\mathscr{D} \downarrow \varnothing$. A Borel measure $\nu$ in $X$ is called $\tau$-additive (weakly $\tau$-additive) if for all $D$ (for $D=\varnothing$ ) $\inf \nu(\mathscr{D})=\nu(D)$ whenever $\mathscr{D} \subseteq \mathscr{F}_{X}$ and $\mathscr{D} \downarrow D$. A completely regular space $X$ is called measure-compact if every Baire measure in $X$ is $\tau$-additive. A topological space $X$ is called Borel measure-compact if every regular Borel measure in $X$ is $\tau$-additive.

Measure-compact and Borel measure-compact spaces have been studied intensively; Wheeler [19] and Gardner-Pfeffer [8] contain a general survey of this subject. Although these properties are defined in terms of measures, they have purely topological nature, that is they are invariant under homeomorphisms. Therefore it is natural to ask about their topological characterizations (cf. [19, Problem 8.13] and [8, p. 994]). Using Corollary 2.10 we get such characterizations in terms of filters and strongly positive families. We follow the terminology introduced at the beginning of $\S 2$.

Theorem 3.1. (a) A completely regular space $X$ is measure-compact if and only if every $\mathscr{Z}_{X}$-filter contained in a $\mathscr{Z}_{X}$-strongly positive family is fixed.

(b) A Hausdorff space $X$ is Borel measure-compact if and only if every $\mathscr{F}_{X}$ filter contained in $\mathscr{F}_{X}$-strongly positive family is fixed.

Lemma 3.2. Let $\mathscr{L}$ be a $\delta$-lattice in an abstract space $X$. The following are equivalent:

(i) for every $\mathscr{L}$-regular measure $\mu$ on $\sigma(\mathscr{L})$, inf $\mu(\mathscr{M})=0$ whenever $\mathscr{M} \subseteq \mathscr{L}$ and $\mathscr{M} \downarrow \varnothing$;

(ii) every $\mathscr{L}$-filter belonging to $\mathrm{sp}^{*}(\mathscr{L})$ is fixed.

Proof. (i) $\Rightarrow$ (ii). If $\mathscr{D}$ is an $\mathscr{L}$-filter such that $\mathscr{D} \in \mathrm{sp}^{*}(\mathscr{L})$ then there exists an $\mathscr{L}$-regular measure $\mu$ with $\left.\mu\right|_{\mathscr{D}}=1$ (Corollary 2.10). Therefore (i) implies that $\cap \mathscr{D} \neq \varnothing$.

(ii) $\Rightarrow$ (i). Suppose that there are an $\mathscr{L}$-regular measure $\mu$ and $\mathscr{M} \subseteq \mathscr{L}$ such that $\mathscr{M} \downarrow \varnothing$ and $\inf \mu(\mathscr{M})>0$. Then the family

$$
\mathscr{D}=\{L \in \mathscr{L}:(\exists M \in \mathscr{M}) M \subseteq L\}
$$

is an $\mathscr{L}$-filter and $\mathscr{D} \in \mathbf{s p}^{*}(\mathscr{L})$ by Theorem 2.9. But $\cap \mathscr{D}=\bigcap \mathscr{M}=\varnothing$, i.e. the filter $\mathscr{D}$ is not fixed.

Proof of Theorem 3.1. Recall that $\mathscr{Z}_{X}$ is a $\delta$-lattice in a completely regular space $X$ and that every Baire measure in $X$ is $\mathscr{Z}_{X}$-regular [8, Theorem 14.2]. Therefore part (a) follows from Lemma 3.2. Since a regular Borel measure being weakly $\tau$-additive is $\tau$-additive [8, Proposition 6.13], Lemma 3.2 implies part (b) as well.

Remarks. 1. Recall that a completely regular space $X$ is called realcompact if every $\sigma$-complete $\mathscr{Z}_{X}$-ultrafilter is fixed (or, equivalently, every 0 -1 Baire measure in $X$ is $\tau$-additive). Clearly $X$ is realcompact if it is measure-compact; it 
is known that the converse does not hold (cf. [19, p. 128]). Since $\sigma$-complete $\mathscr{Z}_{X}$-ultrafilters are $\mathscr{Z}_{X}$-strongly positive (see the remark after Definition 2.1 ), the condition given in part (a) of the last theorem may be regarded as a strengthening of realcompactness that implies measure-compactness.

2. Stronger completeness properties have been considered (cf. [8]). For example, a space $X$ is called weakly Borel measure complete if every Borel measure in $X$ is weakly $\tau$-additive. By Lemma $3.2 X$ is weakly Borel measure complete if and only if every $\mathscr{F}_{X}$-filter belonging to $\operatorname{sp}^{*}\left(\mathscr{B}_{X}\right)$ is fixed (here $\mathscr{B}_{X}=\sigma\left(\mathscr{F}_{X}\right)$ is the Borel $\sigma$-algebra in $X$ ).

3. Let $X$ be a discrete topological space. It is easy to see that $X$ is measurecompact if and only if every Borel measure in $X$ is concentrated on a countable subset of $X$ (equivalently, the cardinality of $X$ is not real-valued measurable, in the sense of Definition 1.11 from [8]). It follows from Corollary 2.10 that $X$ is measure-compact if and only if each $2^{X}$-strongly positive family contains a countable set.

\section{ON MEASURE EXTENSION PROBLEM}

The existence of measure extensions has been investigated by many authors in the following setting.

Given two $\delta$-lattices $\mathscr{K}$ and $\mathscr{L}$ in an abstract set $X$, find conditions under which every $\mathscr{K}$-regular measure can be extended to an $\mathscr{L}$-regular measure defined on $\sigma(\mathscr{L})$.

There have been developed several techniques of constructing regular extensions of measures; see $[10,3,1]$, and also [7, 12] where the problem is studied in the context of group-valued measures ( $\mathscr{K}$ and $\mathscr{L}$ are usually assumed to be lattices only and $\sigma$-additive quasi-measures are discussed). Problems of this type appear quite naturally in topological measure theory; for example, the question which Baire measures have regular Borel extensions is of great interest (cf. $[19, \S 9])$.

In the sequel, we consider two fixed $\delta$-lattices $\mathscr{K}$ and $\mathscr{L}$, where $\mathscr{K} \subseteq$ $\mathscr{L} \subseteq 2^{X}$. We denote by $\mathscr{A}$ and $\mathscr{B}$ the $\sigma$-algebras generated by $\mathscr{K}$ and $\mathscr{L}$, respectively. The language introduced in $\S 2$ gives a combinatorial answer to the above problem.

Theorem 4.1. For a $\mathscr{K}$-regular measure $\mu$ defined on $\mathscr{A}$, the following are equivalent:

(i) $\mu$ can be extended to an $\mathscr{L}$-regular measure on $\mathscr{B}$;

(ii) $\mathscr{K}_{n}=\{K \in \mathscr{K}: \mu(K) \geq 1 / n\} \in \operatorname{sp}^{*}(\mathscr{L})$ for every $n \in \mathbb{N}$.

Proof. (i) $\Rightarrow$ (ii). If $\nu$ is such an extension of $\mu$ then

$$
\mathscr{K}_{n} \subseteq\{L \in \mathscr{L}: \nu(L) \geq 1 / n\} .
$$

Since the second family belongs to $\operatorname{sp}^{*}(\mathscr{L})$ in view of Theorem $2.9($ a), so does $\mathscr{K}_{n}$.

(ii) $\Rightarrow$ (i). Let $\mathscr{K}^{+}=\{K \in \mathscr{K}: \mu(K)>0\}=\bigcup_{n} \mathscr{K}_{n}$. By Theorem 2.9(b) there exists an $\mathscr{L}$-regular measure $\nu$ on $\mathscr{B}$ such that $\nu(K)>0$ for $K \in \mathscr{K}^{+}$. Thus $\mu(K)=0$ for every $K \in \mathscr{K}$ with $\nu(K)=0$. Since $\mu$ is $\mathscr{K}$-regular, it follows that $\mu$ is absolutely continuous with respect to $\left.\nu\right|_{\mathscr{A}}$. By the RadonNikodym theorem there exists an $\mathscr{A}$-measurable function $g: X \rightarrow[0, \infty)$ such 
that $\mu(A)=\left.\int_{A} g d \nu\right|_{\mathscr{A}}$ for any $A \in \mathscr{A}$. The formula $\bar{\mu}(B)=\int_{B} g d \nu$ defines a measure on $\mathscr{B}$ that extends $\mu$. Since $\bar{\mu}$ is absolutely continuous with respect to the $\mathscr{L}$-regular measure $\nu$, it is $\mathscr{L}$-regular as well.

The last theorem, together with Theorem 2.9, gives the following result.

Corollary 4.2. The following are equivalent:

(i) every $\mathscr{K}$-regular measure on $\mathscr{A}$ can be extended to an $\mathscr{L}$-regular measure on $\mathscr{B}$;

(ii) $\operatorname{sp}(\mathscr{K}) \subseteq \mathrm{sp}^{*}(\mathscr{L})$.

This includes a characterization of Mařik spaces. Recall that a completely regular topological space $X$ is called a Mařik space if every Baire measure in $X$ has a regular Borel extension (cf. [19, Definition 9.1]).

Corollary 4.3. $A$ space $X$ is a Mařik space if and only if every $\mathscr{Z}_{X}$-strongly positive family is contained in some $\mathscr{F}_{X}$-strongly positive family.

Of course, Corollary 4.2 only replaces the problem of constructing a measure extension by the problem of finding, for a given $\mathscr{P} \in \operatorname{sp}(\mathscr{K})$, an $\mathscr{L}$-strongly positive family which includes $\mathscr{P}$. There are special cases, however, in which the second task is easy. Recall that $\mathscr{L}$ is called $\mathscr{K}$ countably bounded if for every sequence $\left\{L_{n}\right\} \subseteq \mathscr{L}$ with $L_{n} \downarrow \varnothing$ there is a sequence $\left\{K_{n}\right\} \subseteq \mathscr{K}$ such that $L_{n} \subseteq K_{n}$ and $K_{n} \downarrow \varnothing$.

Corollary 4.4 (Bachmann-Sultan [3, Theorem 2.1]). If $\mathscr{L}$ is $\mathscr{K}$ countably bounded then every $\mathscr{K}$-regular measure on $\mathscr{A}$ admits an $\mathscr{L}$-regular extension to a measure on $\mathscr{B}$.

Proof. In view of Corollary 4.2 it is enough to check that $\operatorname{sp}(\mathscr{K}) \subseteq \operatorname{sp}(\mathscr{L})$. Let $\mathscr{P} \in \operatorname{sp}(\mathscr{K})$. If $L_{n} \subseteq K_{n}$ for every $n$ then

$$
\inf _{n} I\left(\mathscr{P}, L_{n}\right) \leq \inf _{n} I\left(\mathscr{P}, K_{n}\right),
$$

and therefore $\mathscr{P} \in \operatorname{sp}(\mathscr{L})$ by the assumption.

\section{ACKNOWLEDGMENT}

The author wishes to thank Professor Cz. Ryll-Nardzewski, Professor K. Musial and A. Morawiec for several helpful comments concerning this paper.

\section{REFERENCES}

1. W. Adamski, Extensions of tight set functions with applications in topological measure theory, Trans. Amer. Math. Soc. 283 (1984), 353-368.

2. G. Bachmann and R. Cohen, Regular lattice measures and repleteness, Comm. Pure Appl. Math. 26 (1973), 587-599.

3. G. Bachmann and A. Sultan, On regular extensions of measures, Pacific J. Math. 86 (1980), 389-395.

4. K. P. S. Bhaskara Rao and B. V. Rao, Theory of charges, Academic Press, New York, 1983.

5. J. A. Van Casteren, Supports of Borel measures, Univ. Antwerpen, 1974, preprint.

6. W. W. Comfort and S. Negrepontis, Chain conditions in topology, Cambridge Univ. Press, 1982.

7. K. P. Dalgas, A general extension theorem for group-valued measures, Math. Nachr. 106 (1982), 153-170. 
8. R. J. Gardner and W. F. Pfeffer, Borel measures, Handbook of Set-Theoretic Topology, Elsevier, 1984.

9. P. R. Halmos, Measure theory, Van Nostrand, 1950.

10. W. Josephson, Coallocation between lattices with applications to measure extensions, Pacific J. Math. 75 (1978), 149-163.

11. J. L. Kelley, Measures on Boolean algebras, Pacific J. Math. 9 (1959),1165-1177.

12. Z. Lipecki, Tight extensions of group-valued quasi-measures, Colloq. Math. 51 (1987), 213219.

13. J. Lembcke, Konservative Abbildungen und Fortsetzung regulärer Masse, Z. Wahrsch. Verw. Gebiete 15 (1970), 57-96.

14. G. Mägerl and I. Namioka, Intersection numbers and weak* separability of spaces and measures, Math. Ann. 249 (1980), 273-279.

15. E. Marczewski (Szpilrajn), Remarques sur les fonctions completement additives et sur les ensembles jouissant de la propriete de Baire, Fund. Math. 22 (1937), 303-311.

16. E. Marczewski, On compact measures, Fund. Math. 40 (1953), 113-124.

17. J. Pachl, Disintegration and compact measures, Math. Scand. 43 (1978), 157-168.

18. G. Plebanek, On strictly positive measures on topological spaces, Atti Sem. Mat. Fis. Univ. Modena 39 (1991), 181-191.

19. R. F. Wheeler, A survey of Baire measures and strict topologies, Exposition. Math. 2 (1983), 9-190.

20. M. Wilhelm, Existence of additive functional on semigroups and the von Neumann minimax theorem, Colloq. Math. 35 (1976), 268-274.

Institute of Mathematics, Wroclaw University, Pl. Grunwaldzki 2/4, 50384 Wroclaw, PolAND

E-mail address: logicuwr@plwrtu11.bitnet 